\title{
Nephromegaly in Children with Kawasaki Disease: New Supporting Evidence for Diagnosis and Its Possible Mechanism
}

\author{
HSIN-PING HUANG, YI-CHUN LAI, I-JUNG TSAI, SHIH-YU CHEN, CHI-HUI CHENG, AND YONG-KWEI TSAU \\ Department of Pediatrics [Y.-C.L., I.-J.T., S.-Y.C.], National Taiwan University Hospital, Taipei 100, Taiwan; Branch for Women and \\ Children [H.-P.H.], Taipei City Hospital, Taipei 100, Taiwan; Department of Pediatrics [C.-H.C., Y.-K.T.], Chang Gung Children's \\ Hospital, Taoyuan 333, Taiwan
}

\begin{abstract}
To measure the kidney size in children with Kawasaki disease (KD) and to delineate the condition of nephromegaly, 20 children with $\mathrm{KD}$ were enrolled in our study. Kidney sizes were measured during acute stage in these patients. Twenty healthy children and 15 febrile children served as healthy controls and fever controls, respectively. To delineate the possible mechanism, we also evaluated kidney volume (KV) in 13 other patients with KD during the acute phase and after the recovery phase as well as 26 healthy children. Plasma hepatocyte growth factor (HGF) and transforming growth factor- $\beta 1$ (TGF- $\beta 1$ ) levels were determined for all children. Kidney lengths and KV in patients with KD during the acute phase were significantly larger than those of healthy children. There was no kidney enlargement in healthy controls and after the recovery phase. The ratio of patient plasma HGF/TGF- $\beta 1$ during the acute phase and after the recovery phase correlated positively with the degree of nephromegaly in all patients. These results confirm the presence of large kidneys in children with KD. Our data also suggest that an elevated HGF/TGF- $\beta 1$ ratio may be responsible for the transient nephromegaly in these children. (Pediatr Res 63: 207-210, 2008)
\end{abstract}

$\mathrm{K}$ awasaki disease (KD) is an acute generalized vasculitis of unknown etiology occurring mostly in infants and young children. Dr. Tomisaku Kawasaki first reported this disease in 1967 (1) and published the first English report of 50 patients in 1974 (2). The diagnosis is confirmed by the presence of fever for at least $5 \mathrm{~d}$ and four of five classic features: conjunctivitis, lymphadenopathy, polymorphous rash, mucosa change, and extremities change (3). The most important issue in this disease is the correlation with subsequent cardiac complications in some cases. Currently, it is the leading cause of acquired heart disease in children in the developing countries and is reported as a potential risk factor for adult ischemic heart disease $(4,5)$. However, there is no definitive diagnostic laboratory test available. If effective treatment is not given early in the course of the disease, coronary artery aneurysm develops in $20 \%-25 \%$ of cases $(4,6)$.

Sterile pyuria in KD is one of a broad range of nonspecific clinical findings and the cause is unknown (7). This feature can mislead physicians to misdiagnose this disease as a urinary tract infection and lead to a series of workups, including ultrasound

Received June 21, 2007; accepted September 26, 2007.

Correspondence: Yong-Kwei Tsau, M.D., Department of Pediatrics, National Taiwan University Hospital, No.7, Chung-Shan South Road, Taipei 100, Taiwan; e-mail: tsauyk@ntu.edu.tw

This work was supported by grants from the National Science Council of Taiwan (NSC96-2314-B-002-069-MY2). evaluation. The accidental detection of large kidneys during renal ultrasound examination in several patients with KD encouraged us to perform a prospective study to confirm this finding.

We previously showed a positive correlation between the nephromegaly and plasma hepatocyte growth factor (HGF)/ transforming growth factor- $\beta 1$ (TGF- $\beta 1$ ) ratio in children with hepatobiliary diseases $(8-10)$. The reciprocal change between HGF and TGF- $\beta 1$ may suggest a decreased antiproliferative effect of TGF- $\beta 1$ on renal growth and also potentiate the mitogenic action of HGF, leading to nephromegaly. Whether nephromegaly in KD is HGF/TGF- $\beta 1$ ratio-related is unknown. The purpose of this study was to measure renal size in children with $\mathrm{KD}$ and to correlate it with plasma HGF/TGF- $\beta 1$ ratios.

\section{METHODS}

Study design concerning nephromegaly in KD. A total of 20 children (13 boys and seven girls of between the ages of 3 and $78 \mathrm{mo}$ ) with KD at the National Taiwan University Hospital (Taipei, Taiwan) were enrolled in our study from February to June 2006. The diagnosis was established based on standard diagnostic guidelines for KD (2-4), including the absence of an alternative diagnosis. All patients had urine cultures with no growth of bacteria. All patients had normal renal function and no recent history of urinary tract infection. All received renal ultrasound examination to measure kidney sizes before i.v. human immunoglobulin (IVIG) administration.

Twenty age- and sex-matched healthy children served as healthy controls. Fifteen febrile children (10 boys and five girls with ages ranging from 11 to $108 \mathrm{mo}$ ) with active infections (four pneumonia, three scarlet fever, three upper airway infection, two herpangina, one bacterial colitis, one roseola, and one ruptured appendix) served as disease controls. The timing of kidney size measurement in fever controls was on d 4-6 during the fever course.

Study design concerning the mechanism of nephromegaly. Thirteen children who met the diagnostic criteria for KD (2-4) were enrolled in another prospective study from March through September 2006. Twenty-six age- and sex-matched healthy children served as controls. All patients received IVIG at a dose of $1 \mathrm{~g} / \mathrm{kg} / \mathrm{d}$ for $2 \mathrm{~d}$ and subsequent acetylsalicylic acid treatment $(80-100 \mathrm{mg} / \mathrm{kg} / \mathrm{d}$ during the febrile phase followed by $3-5 \mathrm{mg} / \mathrm{kg} / \mathrm{d}$ since defervescence). In children with $\mathrm{KD}$, a renal sonogram and plasma were obtained on d $5.3 \pm 1.6$ of illness in the acute febrile phase before IVIG administration, as well as at 1 mo after defervescence (recovery phase). Absence of fever occurred on $\mathrm{d} 7.7 \pm 1.1$ of illness for these patients.

Renal ultrasonography was performed in all diseased and healthy children to measure renal size. Blood was drawn from each patient for plasma HGF and TGF- $\beta 1$ determination. After centrifugation at $1000 \times \mathrm{g}$, ethylenediamine tetraacetic acid plasma samples were frozen at $-70^{\circ} \mathrm{C}$ until tested.

All studies were performed with informed written parental consent and approved by the Research Committee of National Taiwan University Hospital.

Kidney measurements. All sonograms were obtained by using a commercial unit from GE Medical Systems, model LOGIQ 400 (GE Medical Systems, Milwaukee, WI) with a 5.0-MHz probe. Bilateral longitudinal renal

Abbreviations: HGF, hepatocyte growth factor; IVIG, intravenous human immunoglobulin; KD, Kawasaki disease; KV, kidney volume 
lengths (L) and maximal anteroposterior (AP) and transverse (W) diameters were measured. Kidney volumes (KV) were calculated according to the formula: $\mathrm{KV}=0.523 \times \mathrm{L} \times \mathrm{W} \times \mathrm{AP}$ (11), and only the left kidney length and KV results were discussed. To avoid interobserver errors during the scanning and measurement, all scanning was performed by the same physician who did not know the diagnosis. Normal renal lengths for children in Taiwan were obtained from the Chu et al. report (12). Standard deviation score (SDS) for renal length was calculated according to the formula described previously (13). Absolute nephromegaly was defined as kidney length $>$ mean $+2 \mathrm{SD}$ for age. The degree of nephromegaly was evaluated by means of $\mathrm{KVa} / \mathrm{KVr}$, which indicated the ratio of patient $\mathrm{KV}$ during the acute phase and after the recovery stage of KD.

$\boldsymbol{H G F}$ and TGF- $\boldsymbol{\beta} 1$ assays. HGF concentrations were determined by using an enzyme-linked immunosorbent assay (ELISA) (human HGF kit; R\&D Systems Inc, Minneapolis, MN). We used the ELISA with an monoclonal antibody to recognize both natural and recombinant human HGF. TGF- $\beta 1$ concentrations were measured by using a human TGF- $\beta 1$ kit from R\&D Systems. The assay procedures were conducted according to the manufacturer's instructions, as described previously (8). All samples were measured in duplicate. Intra- and interassay coefficients of variation were $5.6 \%$ and $7.6 \%$, respectively, for HGF, and $5.1 \%$ and $8.0 \%$, respectively, for TGF- $\beta 1$.

Statistical analysis. Data are reported as the mean \pm SD when appropriate. The correlation between renal length and age or between KV and age or HGF/TGF- $\beta 1$ ratio was examined by linear regression analysis. Analysis of covariance was used to examine the difference between the two regression lines. A $t$ test was used to compare KVs. One-way analysis of variance followed by the Bonferroni $t$ test was used for comparison of renal length SDS or HGF/TGF- $\beta 1$ ratios among groups. Statistical significance was set at $p<0.05$.

\section{RESULTS}

Figure 1 illustrates the correlation of left kidney length with age in children with $\mathrm{KD}(y=0.0376 x+6.6497, r=0.864, p<$ $0.001)$ and in the healthy children $(y=0.0385 x+5.6372, r=$ $0.929, p<0.001)$. There was a significantly increased renal length in the children with $\mathrm{KD}(p<0.001)$. Table 1 shows left $\mathrm{KVs}$ in these two groups of children. There was also a significantly increased KV in the children with $\mathrm{KD}(p=0.001)$. Large kidneys in KD were indicated by an increase in both length and volume of the kidney, providing strong evidence of the manifes-

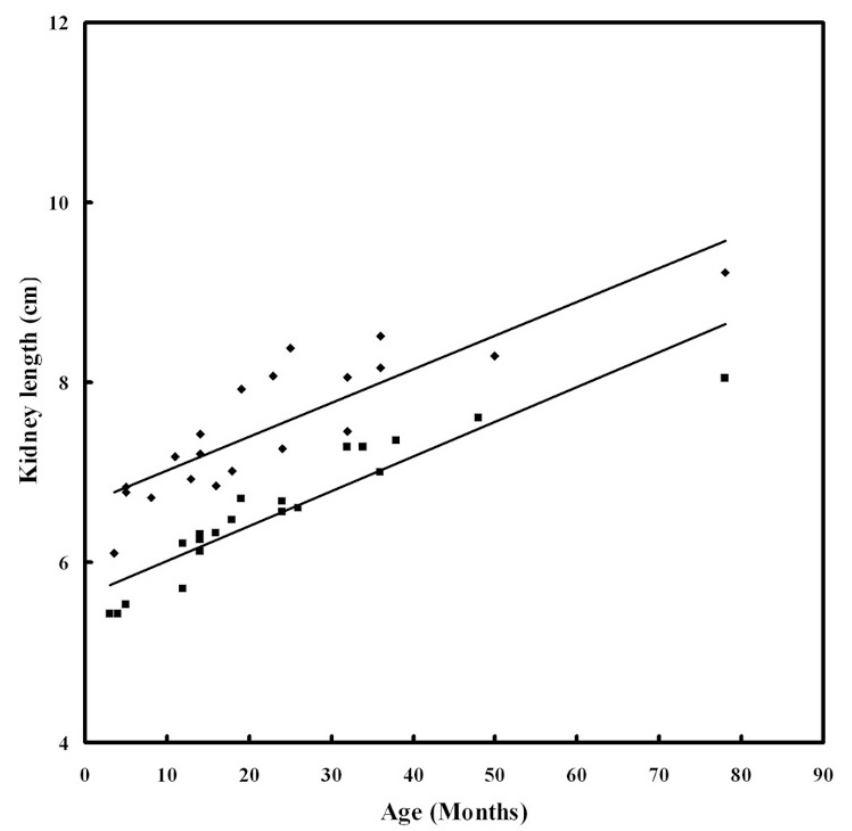

Figure 1. Regression analysis of left renal length in children with KD ( ) and in healthy children (匹). Renal lengths are significantly larger in children with KD than those of healthy children. $(p<0.001$ for the intercept and $p=$ 0.883 for the slope, by analysis of covariance). tation of nephromegaly. Analysis of right kidneys yielded similar results (data not shown).

The renal length SDS in children with KD was significantly higher than that in the fever controls or healthy children $(p<$ 0.001), also indicating nephromegaly in these patients (Table 1). There was no difference between the fever controls and the healthy children. Fourteen (70\%) children with KD had absolute nephromegaly. However, absolute nephromegaly was not found in either fever controls or healthy children.

Common features of KD in our children are shown in Table 2. Absolute nephromegaly is added in this table for comparison. Each feature is listed based on frequency. Polymorphous skin rash is the most common feature followed by desquamation, oral mucosa change, and conjunctivitis. Neck lymphadenopathy and nephromegaly share the same frequency thereafter.

Most children with KD were noted to have nephromegaly during the acute phase and had apparently normal-sized kidneys after the recovery phase. Figure 2 shows the comparison of KV against age in 13 children with KD during the acute phase $(y=6.81 x+43.96, r=0.630, p=0.02)$ and after the recovery phase $(y=5.24 x+24.34, r=0.772, p=0.002)$. Nephromegaly was noted significantly in children with KD during the acute phase compared with healthy children $(p<$ 0.001 for the intercept and $p=0.620$ for the slope, by analysis of covariance). Enlarged KVs returned to normal in these patients after the recovery phase $(p=0.624$ for the intercept and $p=0.577$ for the slope, compared with healthy children).

Plasma HGF levels were markedly elevated in children with KD during the acute phase compared with those after the recovery phase $(2452 \pm 2058 \mathrm{pg} / \mathrm{mL}$ versus $359.6 \pm 53.4$ $\mathrm{pg} / \mathrm{mL}, p=0.003$ by paired $t$ test). On the contrary, plasma TGF- $\beta 1$ levels were lower during the acute phase than those after the recovery phase $(25.8 \pm 12.6 \mathrm{ng} / \mathrm{mL}$ versus $41.4 \pm$ $18.8 \mathrm{ng} / \mathrm{mL}, p=0.017$ by paired $t$ test) in these patients. Plasma HGF/TGF- $\beta 1$ ratios were higher in children with KD during the acute phase than in healthy children and in patients after the recovery phase (Table 3 ). There also was a positive correlation between change (extent of elevation) of plasma HGF/ TGF- $\beta 1$ ratio and degree of nephromegaly $(y=0.0338 x+$ $1.354, r=0.805, p<0.001$; Fig. 3) for these patients.

\section{DISCUSSION}

Although $\mathrm{KD}$ is a multisystemic vasculitis syndrome, renal involvement is seldom mentioned and not the main characteristic of the disease. Acute renal failure has been a rare, but severe renal manifestation associated with KD (14-16). Enlarged kidneys were always reported as a finding of acute renal failure in patients with KD (14,15). In this study, most of our patients had enlarged kidneys, whereas no patients had evidence of acute renal failure (normal serum creatinine for all patients).

$\mathrm{KD}$ is self-limiting, but there is still a $25 \%$ risk of developing serious cardiovascular damage if proper treatment is not given early in the course of the disease $(4,6)$. The symptoms of KD evolve over the first $10 \mathrm{~d}$ of illness and gradually resolve even in the absence of therapy. Because no specific or sensitive laboratory diagnostic test is available, it is a challenge for physicians to distinguish and identify this disease. 
Table 1. Demographic data and renal size of the study population

\begin{tabular}{|c|c|c|c|c|}
\hline & $\mathrm{KD}$ & Healthy children & Fever controls & $p$ value \\
\hline Age, mo & $23.12 \pm 17.78$ & $23.55 \pm 17.63$ & $48.87 \pm 35.76$ & \\
\hline Sex, male/female & $13 / 7$ & $13 / 7$ & $10 / 5$ & \\
\hline $\mathrm{KV}, \mathrm{cm}^{3}$ & $51.83 \pm 17.93$ & $35.62 \pm 11.24$ & & 0.001 \\
\hline Renal length SDS & $2.54 \pm 0.97$ & $-0.23 \pm 0.82$ & $0.15 \pm 0.51$ & $<0.001$ \\
\hline Absolute nephromegaly,* \% & 70 & 0 & 0 & $<0.001$ \\
\hline
\end{tabular}

* Kidney length larger than mean +2 SD for age.

Table 2. Clinical symptoms and signs in 20 children with KD

\begin{tabular}{ll}
\hline \multicolumn{1}{c}{ Symptom/sign } & No. $(\%)$ \\
\hline Fever $\geq 5 \mathrm{~d}$ & $20(100)$ \\
Skin rash & $20(100)$ \\
Conjunctivitis & $18(90)$ \\
Mucosal change & $18(90)$ \\
Extremities desquamation & $18(90)$ \\
Lymphadenopathy & $14(70)$ \\
Extremities induration & $14(70)$ \\
Absolute nephromegaly & $14(70)$ \\
Sterile pyuria & $10(50)$ \\
Erythema at BCG site & $8(40)$ \\
Coronary arteritis & $7(35)$ \\
\hline
\end{tabular}

BCG, bacille Calmette-Guérin.

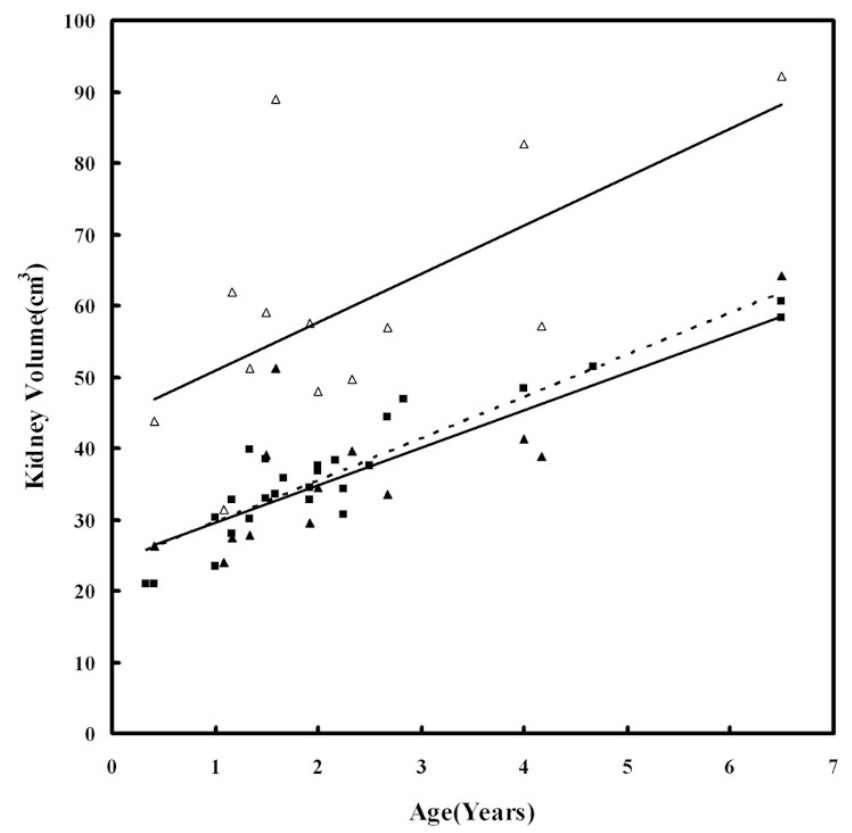

Figure 2. Regression analysis of left KV by age in children with KD during the acute phase $(\triangle)$ and after the recovery phase $(\boldsymbol{\Lambda},---)$, and in healthy controls $(\square,---)$ KVs in patients during the acute phase were significantly higher than those after the recovery phase $(p<0.001$ for the intercept and $p$ $=0.585$ for the slope, by analysis of covariance) and those of healthy children ( $p<0.001$ for the intercept and $p=0.620$ for the slope).

Moreover, children presenting with four manifestations of KD or presenting with coronary arterial lesions plus two to three criteria are considered as cases of atypical $\mathrm{KD}(17,18)$. In this situation, early identification and treatment become difficult, so awareness of other characteristics of KD for physicians may help. In addition to the diagnostic criteria of $\mathrm{KD}$, other features, such as erythema at the bacille Calmette-Guérin (BCG) inoculation site, gallbladder hydrops, high white blood cell count, and platelet count, and low sodium and albumin levels are also mentioned in the literature and could help in the diagnosis of KD $(18,19)$. In our study, up to $70 \%$ of our patients had absolute nephromegaly, occurring as frequently as neck lymphadenopathy and extremities induration, which are two of the five diagnostic criteria for $\mathrm{KD}$, and the incidence of nephromegaly is significantly higher than the appearance of erythema at the BCG site. Nephromegaly in children with KD may be specific given the absence of nephromegaly in our control children with other inflammatory conditions, including three patients with scarlet fever, which is often mistaken for KD clinically.

Nardi et al. (14) speculated that enlarged kidneys in KD might be explained by a vasculitis involving the kidneys with resultant fibrinoid deposits and cellular infiltrations subsequently leading to ischemia followed by edema. However, this proposed mechanism seems unable to explain enlarged kidneys in our patients without renal impairment.

Increased serum HGF levels have been reported previously in children with KD (20). Elevated serum HGF concentration in these patients probably results from increased production of HGF by vascular tissue with inflammation (20). Our results confirm this finding that plasma HGF levels indeed increase in children with KD during the acute stage and return to normal after the recovery stage. These data further encourage us to continue searching for possible HGF/TGF- $\beta 1$ ratio-related mechanisms for nephromegaly in KD.

We previously showed a positive correlation between nephromegaly and plasma HGF/TGF- $\beta 1$ ratios in children with hepatitis or biliary atresia (8-10). This HGF/TGF- $\beta 1$ ratio change has been related to nephromegaly in compensatory renal growth $(9,21)$, and nephromegaly in acute or chronic liver diseases $(8-10,21)$. Although the actual mechanism of HGF/TGF- $\beta 1$ ratio-related nephromegaly is not yet understood, the reciprocal action of HGF and TGF- $\beta 1$ with markedly increased proliferative or renotropic effect (21-24) may explain the resultant nephromegaly. In this study, we demonstrate that nephromegaly is unexpectedly common in patients with KD and it also correlates positively with patient plasma HGF/TGF- $\beta 1$ ratios. This is the first report of HGF/ TGF- $\beta 1$ ratio-related nephromegaly in a disease other than hepatobiliary disorders. It is important to emphasize that this is currently an association in KD. It is possible that both the HGF/TGF- $\beta 1$ and the nephromegaly are markers of disease severity.

Similar to children with fulminant hepatitis (8) or with biliary atresia in liver transplantation (10), nephromegaly in children with KD in the acute stage is also reversible. The change in kidney size is generally in parallel with the change in the plasma HGF/TGF- $\beta 1$ ratio. Thus, our data provide a clue that, similar to children with hepatobiliary diseases, the 
Table 3. $K V$ and laboratory data in 13 children with KD during the acute phase and after the recovery phase

\begin{tabular}{|c|c|c|c|c|}
\hline & \multicolumn{2}{|c|}{ Children with KD } & \multirow{2}{*}{$\begin{array}{l}\text { Healthy } \\
\text { children }\end{array}$} & \multirow[b]{2}{*}{$p$ value } \\
\hline & Acute phase & Recovery phase & & \\
\hline Age, mo & \multicolumn{2}{|c|}{$28.31 \pm 19.82$} & $27.62 \pm 19.07$ & \\
\hline Sex, male/female & \multicolumn{2}{|c|}{$8 / 5$} & $16 / 10$ & \\
\hline $\mathrm{KV}, \mathrm{cm}^{3}$ & $60.03 \pm 17.86$ & $36.71 \pm 11.22$ & $36.9 \pm 10.0$ & $<0.001$ \\
\hline Serum creatinine, $\mathrm{mg} / \mathrm{dL}$ & $0.43 \pm 0.09$ & $0.44 \pm 0.09$ & & 0.368 \\
\hline $\mathrm{HGF}, \mathrm{pg} / \mathrm{mL}$ & $2452 \pm 2058$ & $359.6 \pm 53.4$ & $506.3 \pm 209.1$ & $<0.001$ \\
\hline TGF- $\beta 1, \mathrm{ng} / \mathrm{mL}$ & $25.8 \pm 12.6$ & $41.4 \pm 18.8$ & $38.5 \pm 16.7$ & 0.053 \\
\hline HGF/TGF- $\beta 1, \mathrm{pg} / \mathrm{ng}$ & $101.1 \pm 95.4$ & $11.6 \pm 5.3$ & $15.3 \pm 6.5$ & $<0.001$ \\
\hline
\end{tabular}

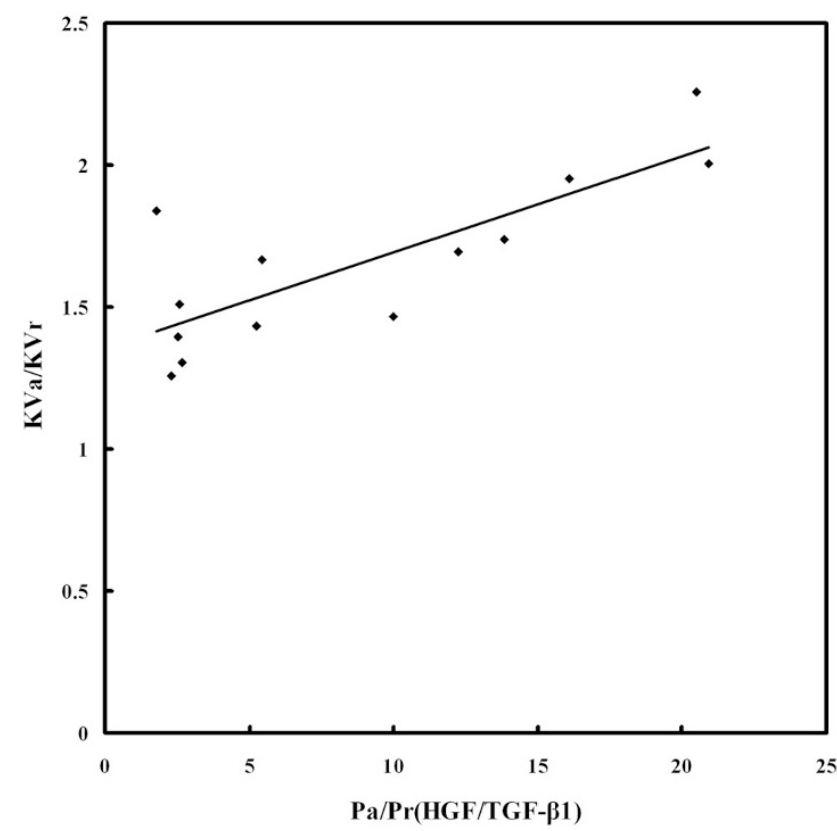

Figure 3. Regression analysis of the degree of nephromegaly on the extent of elevation of the plasma HGF/TGF- $\beta 1$ ratio in children with KD $(r=0.805$, $p<0.001) . \mathrm{KVa} / \mathrm{KVr}$ indicates the ratio of the patient's $\mathrm{KV}$ during the acute phase and after the recovery phase. $\mathrm{Pa} / \mathrm{Pr}(\mathrm{HGF} / \mathrm{TGF}-\beta 1)$ indicates the ratio of the patient's plasma HGF/TGF- $\beta 1$ during the acute phase and after the recovery phase.

plasma HGF/TGF- $\beta 1$ ratio may also be associated with the development of nephromegaly in children with KD.

We are the first to describe nephromegaly in children with $\mathrm{KD}$ who do not have renal impairment. Whether our new findings can offer supporting evidence to aid in the diagnosis of KD remains to be seen. However, we suggest that all children with KD have a renal ultrasound evaluation in the acute stage, especially when the diagnostic criteria are not yet well established. From our data, we also suggest that the plasma HGF/TGF- $\beta 1$ ratio may be associated with the development of nephromegaly, with the degree of resultant nephromegaly being determined by the extent of this ratio change.

\section{REFERENCES}

1. Kawasaki T 1967 [Acute febrile mucocutaneous syndrome with lymphoid involvement with specific desquamation of the fingers and toes in children]. Arerugi $16: 178-222$
2. Kawasaki T, Kosaki F, Osawa S, Shigemitsu I, Yanagawa S 1974 A new infantile acute febrile mucocutaneous lymph node syndrome (MLNS) prevailing in Japan. Pediatrics 54:271-276

3. Morens DM, O'Brien RJ 1978 Kawasaki disease in the United States. J Infect Dis 137:91-93

4. Burns JC, Glode MP 2004 Kawasaki syndrome. Lancet 364:533-544

5. Desai A, Patel S, Book W 2005 'Myocardial infarction' in adolescents: do we have the correct diagnosis? Pediatr Cardiol 26:627-631

6. Newburger JW, Takahashi M, Gerber MA, Gewitz MH, Tani LY, Burns JC, Shulman ST, Bolger AF, Ferrieri P, Baltimore RS, Wilson WR, Baddour LM, Levison ME, Pallasch TJ, Falace DA, Taubert KA Committee on Rheumatic Fever, Endocarditis, and Kawasaki Disease, Council on Cardiovascular Disease in the Young, American Heart Association 2004 Diagnosis, treatment, and long-term management of Kawasaki disease: a statement for health professionals from the Committee of Rheumatic Fever, Endocarditis and Kawasaki disease, Council on Cardiovascular Disease in the Young, American Heart Association. Circulation 110:2747-2771

7. Melish ME, Hicks RM, Larson EJ 1976 Mucocutaneous lymph node syndrome in the Unites States. Am J Dis Child 130:599-607

8. Tsau YK, Lu MY, Ni YH 2001 Nephromegaly and elevated hepatocyte growth factor-transforming growth factor- $\beta 1$ ratio in infants with fulminant hepatitis or biliary atresia. Am J Kidney Dis 38:279-285

9. Tsau YK, Jou ST, Ni YH 2002 Nephromegaly relates to hepatocyte growth factor dysregulation in biliary atresia. Pediatr Nephrol 17:554-559

10. Cheng CH, Tsau YK, Tsai IJ 2005 Kidney volume and plasma hepatocyte growth factor-transforming growth factor $\beta 1$ ratio among children with biliary atresia before and after liver transplantation: the reversibility of nephromegaly. Am J Kidney Dis 46:830-836

11. Hricak H, Lieto RP 1983 Sonographic determination of renal volume. Radiology 148:311-312

12. Chu LW, Lu MY, Tsau YK 1999 Sonographic measurements of renal size in normal children and children with compensatory renal hypertrophy. Acta Paediatr Taiwan 40:18-21

13. Barrett TM, Broyer M, Chantler C, Gilli G, Guest G, Marti-Henneberg C, Preece MA, Rigden SP 1986 Assessment of growth. Am J Kidney Dis 7:340-346

14. Nardi PM, Haller JO, Friedman AP, Slovis TL, Schaffer RM 1985 Renal manifestations of Kawasaki disease. Pediatr Radiol 15:116-118

15. Veiga PA, Pieroni D, Batier W, Feld LG 1992 Association of Kawasaki disease and interstitial nephritis. Pediatr Nephrol 6:421-423

16. Lande MB, Gleeson JG, Sundel RP 1993 Kawasaki disease and acute renal failure. Pediatr Nephrol 7:593-594

17. Burns JC, Wiggins JW, Toews WH, Newburger JW, Leung DY, Wilson H, Glode MP 1986 Clinical spectrum of Kawasaki disease in infants younger than 6 months of age. J Pediatr 109:759-763

18. Ayusawa M, Sonobe T, Uemura S, Ogawa S, Nakamura Y, Kiyosawa N, Ishii M, Harada K. Kawasaki Disease Research Committee 2005 Revision of diagnostic guidelines for Kawasaki disease (the 5th revised edition). Pediatr Int 47:232-234

19. Hsu YH, Wang YH, Hsu WY, Lee YP 1987 Kawasaki disease characterized by erythema and induration at the bacillus Calmette-Guerin and purified protein derivative inoculation sites. Pediatr Infect Dis J 6:576-578

20. Ohno T, Yuge T, Kariyazono H, Igarashi H, Joh-o K, Kinugawa N, Kusuhara K, Hara T 2002 Serum hepatocyte growth factor combined with vascular endothelial growth factor as a predictive indicator for the occurrence of coronary artery lesions in Kawasaki disease. Eur J Pediatr 161:105-111

21. Tsau YK, Tsai IJ, Chen YM 2006 Transient reciprocal change of renal hepatocyte growth factor and transforming growth factor- $\beta 1$ may relate to renal hypertrophy in rats with liver injury or unilateral nephrectomy. Pediatr Res 59:494-499

22. Nagaike M, Hirao S, Tajima H, Noji S, Taniguchi S, Matsumoto K, Nakamura T 1991 Renotropic functions of hepatocyte growth factor in renal regeneration after unilateral nephrectomy. J Biol Chem 266:22781-22784

23. Taipale J, Keski-Oja J 1996 Hepatocyte growth factor releases epithelial and endothelial cells from growth arrest induced by transforming growth factor- $\beta 1$. J Biol Chem 271:4342-4348

24. Matsumoto K, Mizuno S, Nakamura T 2000 Hepatocyte growth factor in renal regeneration, renal disease and potential therapeutics. Curr Opin Nephrol Hypertens 9:395-402 